\title{
ORIGINAL ARTICLE Effects of calcitriol on experimental spinal cord injury in rats
}

\author{
K-1 Zhou ${ }^{1,2}$, D-h Chen ${ }^{1,2}, \mathrm{H}-\mathrm{m}$ Jin $^{1,2}, \mathrm{~K} \mathrm{Wu}^{1,2}, \mathrm{X}-\mathrm{y} \mathrm{Wang}^{1,2}, \mathrm{H}-\mathrm{z} \mathrm{Xu}^{1,2}$ and X-1 Zhang ${ }^{1,2}$
}

Study design: Experimental, controlled, animal study.

Objectives: To evaluate the effects of calcitriol on oxidative stress, apoptosis, autophagy and locomotor recovery in rats after spinal cord injury (SCl).

Setting: China.

Methods: Ninety female rats were randomly divided into three groups. Laminectomy only was performed in the control group. The $\mathrm{SCl}$ group received laminectomy as well as spinal cord compression injury. In the calcitriol group, SCl rats received an intraperitoneal injection of calcitriol $\left(2 \mu \mathrm{g} \mathrm{kg}^{-1}\right.$ day $\left.^{-1}\right)$. Oxidative stress was assessed by the tissue superoxide dismutase (SOD) activity and the contents of glutathione (GSH) and malondialdehyde (MDA). The extent of apoptosis was assessed by immunohistochemistry for C-caspase3, TUNEL staining and western blotting for C-caspase3, Bax and Bcl2. Transmission electron microscopy was used to examine autophagosomes in the injured spinal cord of calcitriol-treated rats. Autophagy was detected by western blotting for LC3-II, Beclin1 and p62. Histological changes were assessed by haematoxylin and eosin staining and Nissl staining. Functional recovery was reflected by the Basso, Beattie and Bresnahan locomotion rating scale and the inclined plane test.

Results: With calcitriol treatment, oxidative stress was decreased, SOD activity and GSH content were increased and MDA content was decreased. Moreover, apoptosis was inhibited in the SCI plus calcitriol group. However, a higher level of autophagy was detected in the lesions of the calcitriol group compared with the $\mathrm{SCl}$ group. Histological damage and neuron loss after $\mathrm{SCl}$ were reduced in calcitrioltreated rats, and functional recovery was significantly promoted in the calcitriol group compared with controls.

Conclusions: Calcitriol promotes locomotor recovery after $\mathrm{SCl}$ by reducing oxidative stress and inhibiting apoptosis, as well as promoting autophagy.

Spinal Cord (2016) 54, 510-516; doi:10.1038/sc.2015.217; published online 5 January 2016

\section{INTRODUCTION}

Spinal cord injury (SCI) is a serious health problem, which places enormous psychological pressure and financial burden on patients' families as well as on society. ${ }^{1}$ Studies have shown that the pathological course of SCI includes primary injury, that is, the direct mechanical effects on the spine, and secondary injury involving a complex cascade of molecular events, including disturbances in ion homeostasis, local oedema, focal haemorrhage, excitotoxicity, inflammatory responses and free radical stress. ${ }^{2}$ Furthermore, oxidative stress and apoptosis were shown to be important factors precipitating posttraumatic degeneration due to secondary destruction of the damaged spinal cord. ${ }^{3}$ Although the precise molecular pathway of secondary injury is still controversial, therapeutic strategies that inhibit or delay oxidative stress and apoptosis may contribute to SCI recovery.

Autophagy, a process used by cells to degrade cytosolic macromolecules and organelles in lysosomes, is generally considered a survival tactic to protect cells under conditions of stress (e.g., starvation, prooxidant conditions). ${ }^{4}$ Autophagy, which is a specific type of programmed cell death, is closely associated with, and shares some molecular events and regulators with, apoptosis. ${ }^{5}$ Recent evidence suggests a strong link between autophagy and SCI.
Kanno $\mathrm{H}$ et al. ${ }^{6}$ first reported that autophagy is activated in the injured spinal cord. Furthermore, recent evidence suggests that stimulation of autophagy after SCI contributes to nerve cell selfprotection and locomotive recovery. ${ }^{7}$

Calcitriol $\left(\mathrm{C}_{44} \mathrm{H}_{80} \mathrm{O}_{4} \mathrm{Si}_{2}\right.$, also known as 1,25-dihydroxyvitamin $\left.\mathrm{D}_{3}\right)$, which has biological functions in bone and calcium metabolism, has a key role in osteoporosis. However, calcitriol has recently been shown to have a number of bioactivities. Recent studies indicated that calcitriol can depress oxidative stress status and prevent oxidative damage in renal ischaemia-reperfusion injury. ${ }^{8}$ Calcitriol attenuates levodopa-induced neurotoxicity in neural stem cells by significantly reducing apoptosis. ${ }^{9}$ In addition, calcitriol has been reported to upregulate the level of cellular autophagy. ${ }^{10}$ However, its relevance to promotion of functional recovery after SCI in rats has not been reported. In the present study, the effects of calcitriol on SCI and the underlying mechanism of action were explored.

\section{MATERIALS AND METHODS}

Animal preparations

Adult female Sprague-Dawley rats weighing 220-250 g were purchased from the Wenzhou Medical University (Wenzhou, China, SCXK[Zhe] 2005-0019).

${ }^{1}$ Department of Orthopaedics, The Second Affiliated Hospital of Wenzhou Medical University, Wenzhou, Zhejiang Province, China and ${ }^{2}$ Zhejiang Provincial Key Laboratory of Orthopaedics, Wenzhou, Zhejiang Province, China

Correspondence: Professor H-z Xu or Dr X-I Zhang, Zhejiang Provincial Key Laboratory of Orthopaedics, The Second Affiliated Hospital of Wenzhou Medical University, No. 109, XueYuan Road (West), LuCheng District, Wenzhou 325000, Zhejiang, China.

E-mail: spinexu@163.com or callmeroger@126.com

Received 6 August 2015; revised 25 October 2015; accepted 6 November 2015; published online 5 January 2016 
Animals were housed in a specific pathogen-free room with a $12 \mathrm{~h}$ light/dark cycle and fed with food and water regularly. The protocol for animal care and use conformed to the Guide for the Care and Use of Laboratory Animals from the National Institutes of Health and was approved by the Animal Care and Use Committee of Wenzhou Medical University (wydw2014-0129). All rats were randomly divided into three groups: a control group, an SCI group and a calcitriol group. Each group contained 30 rats.

\section{Spinal cord injury}

All animals in the three groups were anaesthetised using 2\% pentobarbital sodium (40 $\mathrm{m} \mathrm{kg}^{-1}$; Solarbio Science \& Technology, Beijing, China) by intraperitoneal injections. During the procedures, the temperature of the animals was recorded and maintained at $37^{\circ} \mathrm{C}$ with a heating pad. The vertebral column was exposed and a laminectomy was carried out carefully at the T9 level and then compressed with a vascular clip (15 g force; Oscar, China) for $1 \mathrm{~min}$; the exposed spinal cord was subjected to crushing injury. Rats in the control group were given the same surgical procedure, except compression injury, and the spinal cord was exposed for $1 \mathrm{~min}$. After the SCI, manual urinary bladder emptying was performed three times daily until the return of the bladder function.

\section{Drug administration}

Calcitriol (Cayman, Ann Arbor, MI, USA) was dissolved in ethanol $\left(1 \mathrm{mg} \mathrm{ml}^{-1}\right)$ and further diluted in saline immediately prior to intraperitoneal (i.p.) administration. The calcitriol group $(n=30)$ received calcitriol $2 \mu \mathrm{g} \mathrm{kg}^{-1} \mathrm{day}^{-1}$ on 7 consecutive days. The control group $(n=30)$ and the SCI group $(n=30)$ received the equal amount of saline supplemented with the required volume of ethanol during 7 days. The animals received the first drug solution $2 \mathrm{~h}$ after the surgical procedure.

\section{The Basso, Beattie and Bresnahan locomotion rating scale scores} The Basso, Beattie and Bresnahan (BBB) locomotion rating scale scores ${ }^{11}$ were used for the assessment of locomotion recovery of rats in different groups. Three, 7, 14, 21 and 28 days after the SCI, rats ( $n=6$ per group) were laid on the floor, and crawling ability was observed for $5 \mathrm{~min}$. Outcome measures were obtained in a blinded manner by independent examiners and averaged. In brief, the $\mathrm{BBB}$ scores range from 0 points, which indicates complete paralysis, to 21 points, which indicates normal locomotion.

\section{The inclined plane test}

The inclined plane test was performed via a method described before. ${ }^{12}$ In brief, all rats ( $n=6$ per group) were tested in two positions (right side or left side up) on a testing apparatus (i.e., a board covered with a rubber mat containing horizontal ridges that were spaced $3 \mathrm{~mm}$ apart). For each position, the maximum angle at which a rat could maintain its position for $5 \mathrm{~s}$ without falling was recorded and averaged to obtain a single score for each rat.

\section{Haematoxylin-eosin staining}

Rats ( $n=6$ per group) were re-anaesthetised with $2 \%(\mathrm{w} / \mathrm{v})$ pentobarbital sodium ( $40 \mathrm{mg} \mathrm{kg}^{-1}$, i.p.) and perfused with normal saline, followed by $4 \%(\mathrm{w} / \mathrm{v})$ paraformaldehyde in phosphate-buffered saline on the seventh day after surgery. Spinal cord segments $(1 \mathrm{~mm}$ in length), which spanned a $3 \mathrm{~mm}$ length in the spinal cord centred at the epicentre, were collected and post-fixed in $4 \%(\mathrm{w} / \mathrm{v})$ paraformaldehyde for $24 \mathrm{~h}$. Then, samples in each group were embedded in paraffin for transverse paraffin sections. The paraffin sections $(4 \mu \mathrm{m}$ thick) mounted on poly-L-lysine-coated slides for histopathological examination by haematoxylin and eosin staining. In the end, HE staining was observed under light microscopy (Olympus, Tokyo, Japan).

\section{Nissl staining}

Spinal cord tissues ( $1 \mathrm{~mm}$ segments; $n=6$ per group) were excised and fixed, as before. The samples were embedded in paraffin for coronal paraffin sections. Paraffin sections ( $4 \mu \mathrm{m}$ thick) were mounted on poly-L-lysine-coated slides for Nissl staining; coronal paraffin sections were deparaffinised with xylene and rehydrated through graded ethanol. After washing, the sections were blocked with $3 \%(\mathrm{v} / \mathrm{v}) \mathrm{H}_{2} \mathrm{O}_{2}$ and treated with $10.2 \mathrm{mmoll}^{-1}$ sodium citrate buffer for antigen retrieval for $20 \mathrm{~min}$ at $95^{\circ} \mathrm{C}$. After washing, sections were incubated in $1 \%(\mathrm{w} / \mathrm{v})$ cresyl violet for Nissl staining and observed under a light microscope (Olympus). The Nissl-positive cells were automatically counted at six randomly selected fields from the lesion site per sample by using the Image-Pro Plus (IPP) software, version 6.0 (Media Cybernetics, Rockville, MD, USA).

\section{Immunohistochemistry}

Spinal cord tissues ( $1 \mathrm{~mm}$ segments; $n=6$ per group) were excised and fixed, as before. The samples were embedded in paraffin for coronal paraffin sections. Paraffin sections ( $4 \mu \mathrm{m}$ thick) were mounted on poly-L-lysine-coated slides for immunohistochemistry; coronal paraffin sections were deparaffinised with xylene and rehydrated through graded ethanol. After washing, the sections were blocked with $3 \%(\mathrm{v} / \mathrm{v}) \mathrm{H}_{2} \mathrm{O}_{2}$ and treated with $10.2 \mathrm{mmoll}^{-1}$ sodium citrate buffer for antigen retrieval for $20 \mathrm{~min}$ at $95^{\circ} \mathrm{C}$. After blocking in $10 \%(\mathrm{v} / \mathrm{v})$ bovine serum albumin dissolved in PBS for $30 \mathrm{~min}$, sections were incubated with antibodies against Cleaved-caspase3 (C-caspase3; 1:500; Cell Signal Technology, Danvers, MA, USA) overnight at $4{ }^{\circ} \mathrm{C}$. Next, sections were incubated with horseradish peroxidase-conjugated secondary antibodies for $2 \mathrm{~h}$ at $37^{\circ} \mathrm{C}$, developed with $\mathrm{DAB}$ and counterstained with haematoxylin. Images were captured at $\times 400$ magnification under a light microscope (Olympus). The positive cells of C-caspase 3 were counted automatically at six randomly selected fields from the lesion site per sample by using the IPP software.

\section{TdT-mediated dUTP biotin nick-end labelling (TUNEL)}

TUNEL was used to detect DNA fragmentation due to apoptotic signalling cascades. Coronal sections ( $n=6$ per group) were washed with distilled water, incubated with proteinase $\mathrm{K}$ for $20 \mathrm{~min}$ at $37^{\circ} \mathrm{C}$ and analysed using the In Situ Cell Death Detection Kit (Roche Molecular Biochemicals, Mannheim, Germany) according to the manufacturer's instructions. Images were visualised using a fluorescence microscope (Olympus) at $\times 100$ magnification, and the percentage of positive cells was quantified using five randomly selected fields per sample.

\section{Transmission electron microscopy}

After fixation in $2.5 \%(\mathrm{w} / \mathrm{v})$ glutaraldehyde overnight, spinal cord tissues were post-fixed in $2 \%(\mathrm{v} / \mathrm{v})$ osmium tetroxide and blocked with $2 \%(\mathrm{v} / \mathrm{v})$ uranyl acetate. Following dehydration in a series of acetone washes, tissues were embedded in Araldite for coronal sections. Semithin section and toluidine blue staining were performed for observation of location. Finally, ultra-thin sections of at least three blocks per sample were cut and observed using Hitachi transmission electron microscopy.

\section{Evaluation of superoxide dismutase activity and content of malondialdehyde and glutathione}

Superoxide dismutase (SOD), malondialdehyde (MDA) and glutathione (GSH) testing kits (Nanjing Jiancheng Biology Institution, Nanjing, China) were used to detect the oxidative stress status of the injured spinal cord. On day 7 postoperatively, spinal cord tissues $(n=6)$ were obtained from the lesion epicentre (2.5 mm cephalad and caudal), weighed, homogenised and diluted to $10 \%(\mathrm{vol} / \mathrm{vol})$ in an ice bath. The homogenate was then centrifuged at 3500 r.p. $\mathrm{m}$. for $10 \mathrm{~min}$. Supernatant solution was collected to determine the following biochemical indices. SOD activity was detected using xanthine oxidase: homogenate $(0.1 \mathrm{ml})$ was mixed with $1.4 \mathrm{ml}$ xanthine oxidase and maintained in a water bath at $37^{\circ} \mathrm{C}$ for $40 \mathrm{~min}$. Developer $(2.0 \mathrm{ml})$ was added for shade selection at $550 \mathrm{~nm}$ following a $10 \mathrm{~min}$ incubation at room temperature. MDA content was detected using modified thiobarbituric acid: homogenate $(0.1 \mathrm{ml})$ was mixed with $0.1 \mathrm{ml}$ dehydrated alcohol, $0.1 \mathrm{ml}$ thiobarbituric acid and $4.0 \mathrm{ml}$ developer, maintained in a water bath at $95^{\circ} \mathrm{C}$ for $40 \mathrm{~min}$, cooled in running water and centrifuged at 4000 r.p.m. for $10 \mathrm{~min}$. The supernatant was obtained, and the absorbance was measured at $532 \mathrm{~nm}$. GSH content was detected using modified dithiobisnitrobenzoicacid: homogenate $(0.5 \mathrm{ml})$ was mixed with $2 \mathrm{ml}$ dithiobisnitrobenzoicacid and centrifuged at 4000 for $10 \mathrm{~min}$. The supernatant $(1 \mathrm{ml})$ was obtained. After that, developer $(1.55 \mathrm{ml})$ was added for shade selection at $420 \mathrm{~nm}$ following a $5 \mathrm{~min}$ incubation at room temperature. 


\section{Western blot analysis}

Total proteins from spinal cord tissues were purified using protein extraction reagents. Rat spinal cord segments $(8 \mathrm{~mm}$; containing the injury epicentre) ( $n=6$ per group) were dissected on day 7 following SCI. The equivalent of $60 \mu \mathrm{g}$ protein was separated on a $12 \%(\mathrm{w} / \mathrm{v})$ gel and transferred onto a PVDF membrane (Bio-Rad Laboratories, Hercules, CA, USA). After blocking with 5\% $(\mathrm{w} / \mathrm{v})$ non-fat milk for $2 \mathrm{~h}$, the membranes were incubated with the following antibodies: Bax, C-caspase3, Bcl2, Beclin1, p62 and GAPDH (1:1000; Cell Signaling Technology, Danvers, MA, USA), LC3 (1:500; Cell Signaling Technology). Next, the membranes were incubated with a goat-anti-rabbit secondary antibody for $2 \mathrm{~h}$ at room temperature, and bands were detected using the enhanced chemiluminescence (ECL) kit (PerkinElmer, Waltham, MA, USA). Band intensity was quantified using the Image Lab 3.0 software (Bio-Rad).

\section{Statistical analysis}

Statistical analyses were carried out using the SPSS.20 statistical software. All values are presented as the means \pm standard error of the mean (s.e.m.). Statistical evaluation of the data was performed by one-way analysis of variance followed by a post hoc comparison test using the least significant difference (equal variances assumed) or Dunnett's T3 (equal variances not assumed) method. $P$-values $<0.05$ were considered to indicate statistical significance.

\section{RESULTS}

\section{Calcitriol attenuated oxidative stress in rats with SCI}

SOD activity and the contents of MDA and GSH were measured to verify the antioxidant effects of calcitriol treatment 7 days after injury. Mean SOD activity and mean contents of MDA and GSH in each group ( $n=6$ per group) were compared. In the rat spinal cord, the mean SOD activity and the mean level of GSH were decreased, whereas the mean MDA content was increased, at 7 days after injury. The calcitriol group had a much higher mean SOD activity $(80.33 \pm 12.37$ $\mathrm{U} \mathrm{mg}^{-1}$ protein ${ }^{-1}$ ) compared with the SCI group $(53.33 \pm 9.07 \mathrm{U}-$ $\mathrm{mg}^{-1}$ protein $^{-1} ; \mathrm{P}<0.05$; Figure 1a). In addition, the mean GSH content in the calcitriol group was $6.98 \pm 1.99 \mathrm{nmol} \mathrm{mg}^{-1}$ protein $^{-1}$ and that in the control group was $5.88 \pm 1.77 \mathrm{nmol} \mathrm{mg}^{-1}$ protein $^{-1}$ $(P<0.01)$ (Figure 1b). However, mean MDA content in the calcitriol group was $6.03 \pm 0.55 \mathrm{nmol} \mathrm{mg}^{-1}$ protein $^{-1}$, which was significantly less than the value of $6.79 \pm 0.39 \mathrm{nmol} \mathrm{mg}^{-1}$ protein $^{-1}$ in the SCI group $(P<0.05$; Figure 1c).

\section{Calcitriol inhibited apoptosis in SCI rats}

To assess the role of apoptosis in the injured spinal cord, TUNEL staining and immunohistochemistry were performed in each group. The green dots were regarded as TUNEL-positive cells (Figure 2a).
No TUNEL-positive cells were detected in the lesions of the sham control group. In contrast, the SCI group showed a significant number of apoptosis-positive cells, with decreases seen in the calcitriol group $(P<0.05$; Figure 2c). Immunohistochemical analysis of apoptosis in SCI revealed significant decreases in the number of C-caspase3positive cells in calcitriol-treated rats compared with SCI rats $(P<0.05$; Figures $2 \mathrm{~b}$ and $\mathrm{d})$. To quantify the level of apoptosis in the spinal cords of the three groups at 7 days after the operation, C-caspase3, Bax and Bcl2 expression levels were examined by western blotting analysis (Figure 2e). Compared with the SCI group, C-caspase 3 and Bax expression levels were downregulated in the injured spinal cord of the calcitriol group $(P<0.05$; Figures $2 \mathrm{f}$ and $\mathrm{g})$. Administration of calcitriol after SCI significantly increased Bcl2 levels $(P<0.05$; Figure 2h).

\section{Calcitriol upregulated autophagy in the injured spinal cord}

We used transmission electron microscopy to observe autophagosomes-double membrane structures containing parts of cytoplasmic organelles-in the spinal cords of calcitriol rats (Figure 3a). To evaluate the effect of calcitriol treatment on the level of autophagy after SCI, we examined the levels of autophagyassociated protein expression at 7 days postoperatively by western blot analysis (Figure 3b). LC3-II, Beclin1 and p62 proteins are biomarkers for determining the level of cell autophagy. Administration of calcitriol after SCI significantly increased LC3-II and Beclin1 levels compared with the SCI group $(P<0.05$; Figures $3 \mathrm{c}$ and $\mathrm{d})$. However, calcitriol treatment significantly reduced p62 expression $(P<0.05$; Figure $3 e)$.

\section{Calcitriol reduced histological damage after SCI}

Differences in histomorphology between the control, SCI and calcitriol groups were analysed by haematoxylin and eosin staining and Nissl staining 7 days after contusion. Progressive destruction of the dorsal white matter and central grey matter was evident in SCI rats relative to the control group, indicative of SCI. Comparisons between the SCI and calcitriol groups revealed significant beneficial effects, including decreased neuron loss and a smaller cavity involving the lateral funiculi, dorsal and central grey matter, in the calcitriol group (Figure 4a). Direct analysis of neuronal survival in the spinal cord was performed using Nissl staining 7 days after injury (Figure $4 \mathrm{~b}$ ). There were significantly fewer Nissl staining-positive cells in the spinal cord lesions of SCI rats. Furthermore, a smaller degree of loss of positively stained neurons was found in the lesions of a

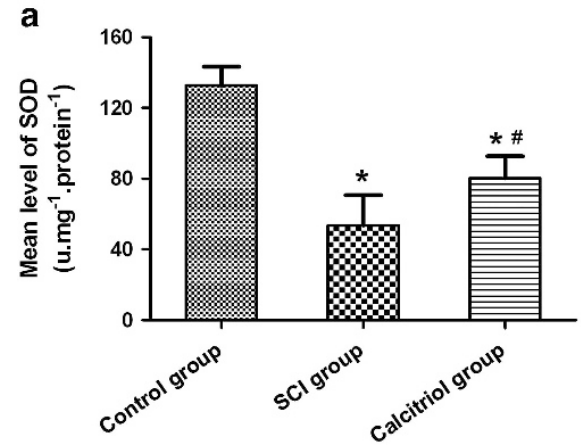

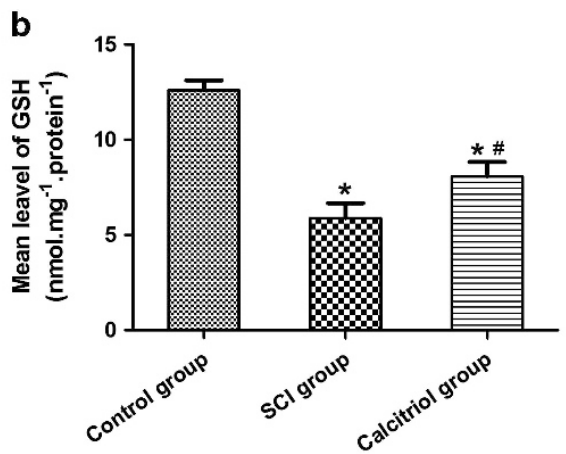

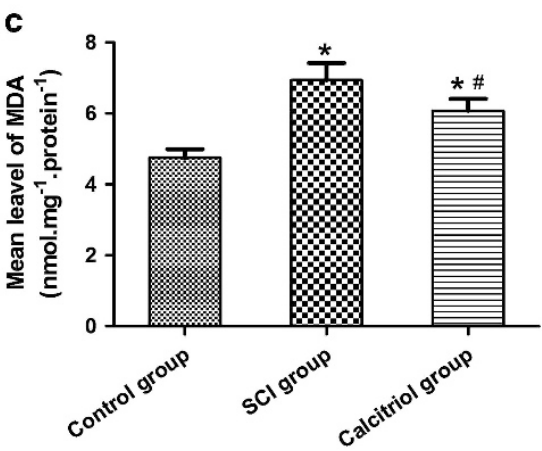

Figure 1 Calcitriol attenuated oxidative stress in rats with spinal cord injury (SCI). (a) The bar graph shows the mean level of superoxide dismutase (SOD) activity in spinal cord lesions in Control, SCl and Calcitriol groups. (b) The bar graph shows mean glutathione (GSH) content in the spinal cord lesions of the three groups. (c) The bar graph shows the mean malondialdehyde (MDA) levels in spinal cord lesions in the three groups. Data are expressed as means \pm s.e.m. ( $n=6$ per group. ${ }^{*} P<0.05$ vs control group, $\# P<0.05$ vs $\mathrm{SCl}$ group; The least significant difference test). 
a
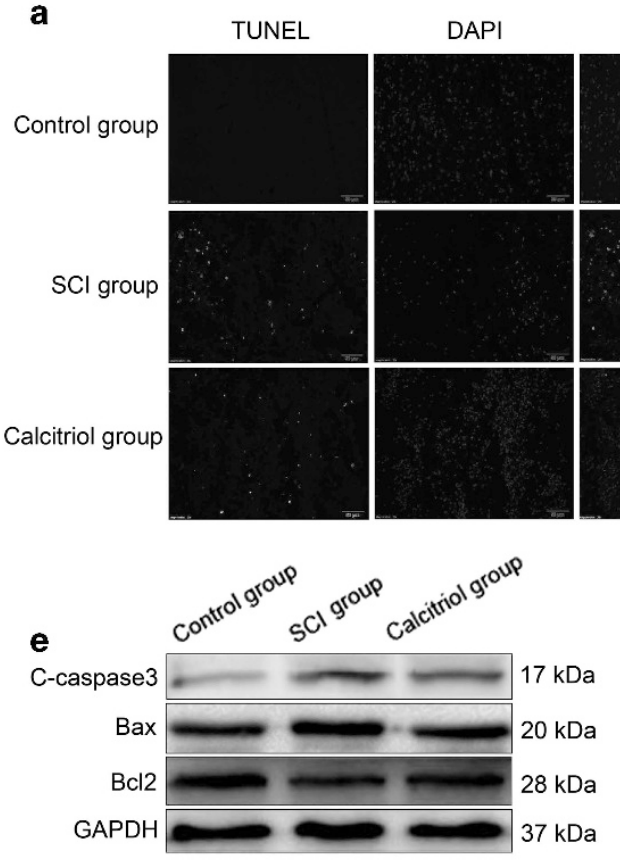

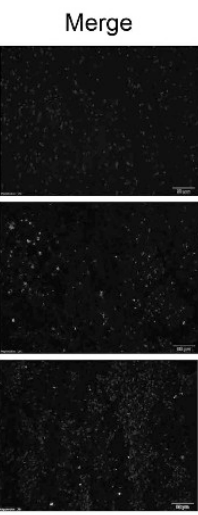

f

b

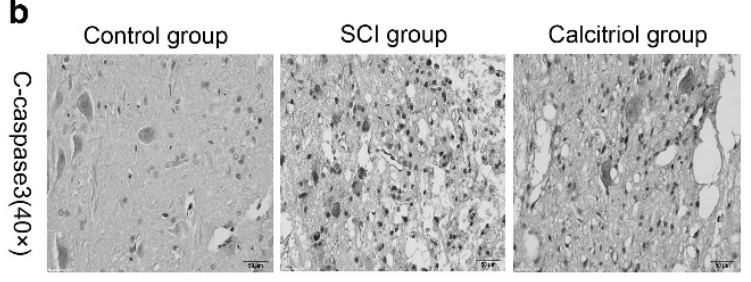

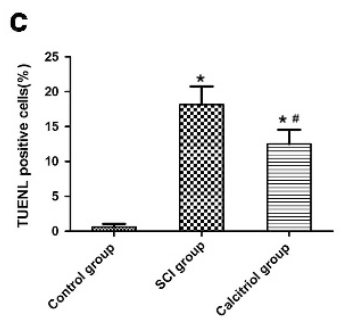

g

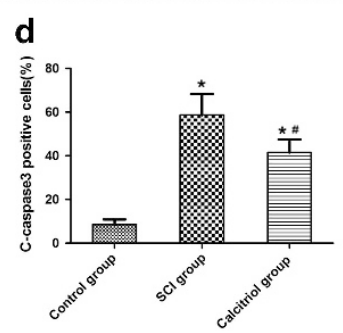

h
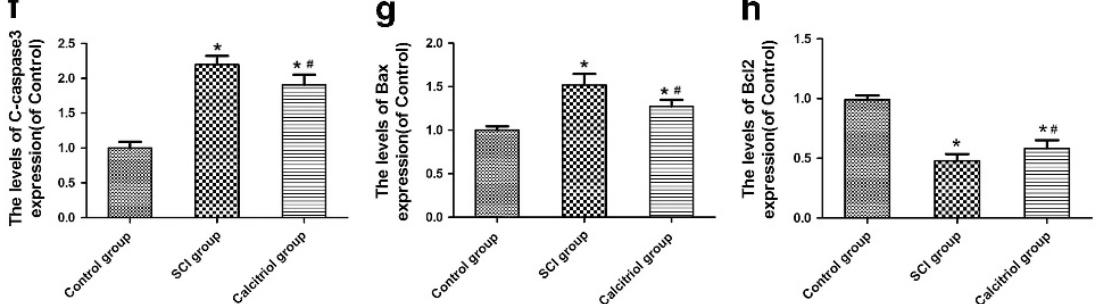

Figure 2 Calcitriol inhibited apoptosis in SCl rats. (a) Representative immunofluorescent TUNEL (green) staining sections from the injured spinal cord in the Control group, the SCI group and the Calcitriol group. Nuclei are labelled with DAPI (blue) (original magnification $\times 200$ ). (b) Immunohistochemistry for C-caspase 3 in the three groups on day 7 after SCl (original magnification $\times 400$ ). (c) The bar graph shows the numbers of TUNEL-positive cells in spinal cord lesions. (d) The bar graph shows the numbers of C-caspase3-positive cells in spinal cord lesions. (e) Protein expression of cleaved caspase3, Bcl2 and Bax in the injured spinal cords of the three groups. GAPDH was used as a loading control and for normalisation of band density. ( $\mathrm{f}-\mathrm{h}$ ) Optical density analysis of C-caspase3, Bcl2 and Bax proteins in the three groups. Data are expressed as means \pm s.e.m. ( $n=6$ per group. * $P<0.05$ vs control group, ${ }^{\#} P<0.05$ vs $\mathrm{SCl}$ group; The least significant difference test).

calcitriol-treated SCI rats compared with the SCI group $(P<0.05$; Figure 4c).

\section{Calcitriol improved locomotor functional recovery of rats receiving $\mathrm{SCI}$}

To evaluate the effects of calcitriol on locomotor functional recovery in rats after SCI, BBB scores and the angle of incline were measured on days 3, 7, 14, 21 and 28 postoperatively. As shown in Figure 5a, the differences in BBB scores between the SCI group and the calcitriol group on days 3 and 7 after SCI were not significant $(P>0.05)$. However, BBB scores were significantly increased in calcitriol-treated rats on days 14, 21 and 28 after SCI compared with the SCI rats $(P<0.05)$. As shown in Figure 5b, calcitriol significantly increased the angle of incline in the inclined plane test on days 7, 14, 21 and 28 days postoperatively. There was no significant difference in the BBB score on day 3 after SCI between the SCI group and the calcitriol group $(P>0.05)$.

\section{DISCUSSION}

In the present study, the angle of incline in the calcitriol group on day 7 was already significantly higher than that in the SCI alone group, whereas the BBB scores remained the same. The mild functional recovery could not be consistently observed in the early days of SCI, which is due to the characteristics of the BBB score and the inclined plane test. BBB scores could be used to assess the locations of claws, movement of the tail and other tiny movements of rat hindlimbs. The inclined plane test mainly focuses on assessment of myodynamia recovery in paraplegic rats. To more accurately assess the improvement of functional recovery in SCI after calcitriol treatment, a long-standing functional assessment was performed based on both the BBB score and the inclined plane test. In addition, we concluded that calcitriol significantly improves locomotor recovery after SCI in rats. The results of haematoxylin and eosin staining and Nissl staining indicated that calcitriol groups exhibited decreased neuron loss and a smaller cavity involving the lateral funiculi, as well as dorsal and central grey matter, after SCI-that is, the functional and histological recovery after SCI in rats showed significant improvements after treatment with calcitriol in the present study. These observations raise questions regarding the biological mechanism underlying the effect of calcitriol treatment in experimental SCI.

The pathological course of SCI includes both primary and secondary injury ${ }^{13}$ The primary injury to the spinal cord is followed by a long period of secondary injury. The levels of oxidative stress and apoptosis are believed to be two of the most important factors involved in post-traumatic degeneration due to destruction of the damaged spinal cord. ${ }^{3}$ Therefore, decreasing oxidation stress injury and inhibition of apoptosis are key factors in the therapeutic schedule of SCI. Calcitriol, that is 1,25-dihydroxyvitamin $\mathrm{D}_{3}$, is the biologically active metabolite of vitamin $\mathrm{D}$ and the predominant $\mathrm{Ca}^{2+}$-regulatory steroid hormone, ${ }^{14}$ which has a major role in osteoporosis. However, increasing numbers of studies indicate that calcitriol has many additional bioactivities. It was reported that calcitriol can significantly decrease apoptosis in levodopa-induced neural stem cell injury. ${ }^{9}$ In addition, similar therapeutic effects were confirmed in an in vitro model of Parkinson's disease. ${ }^{10}$ Therefore, calcitriol treatment reduces 
a
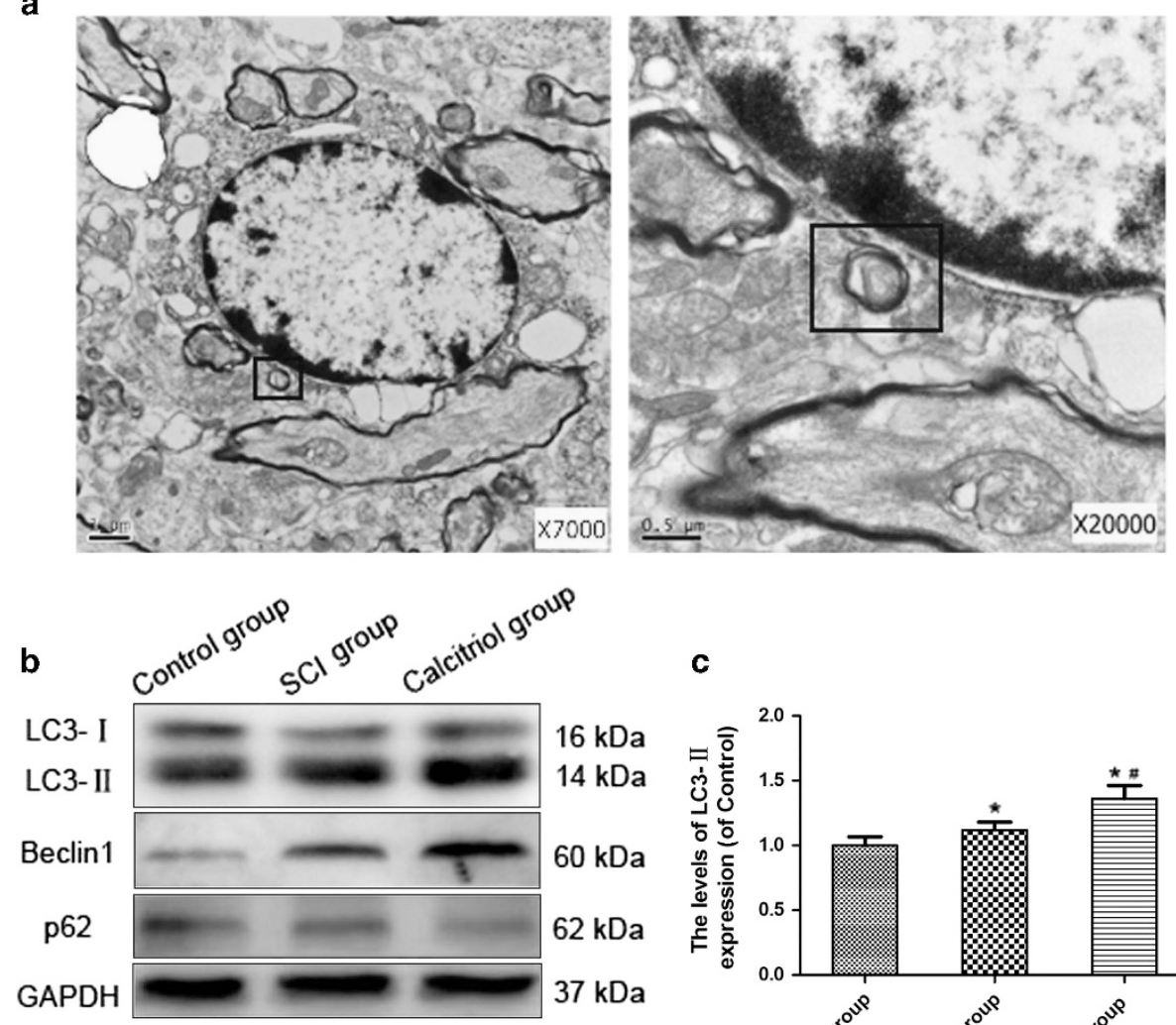

c

d

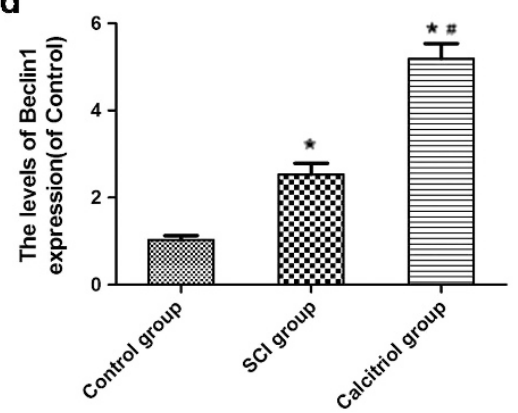

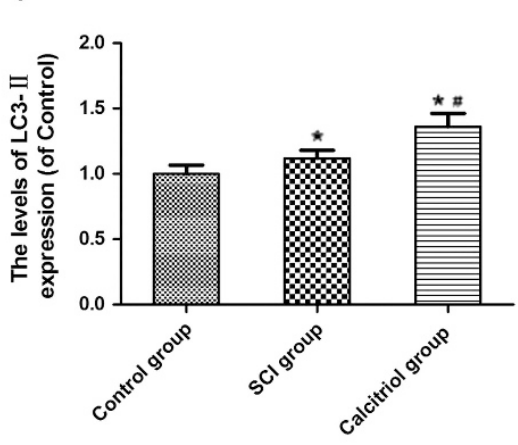

e

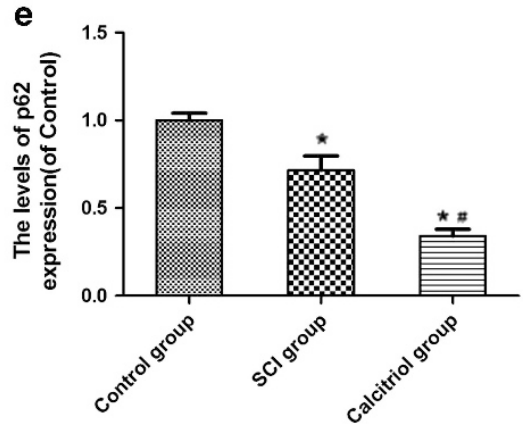

Figure 3 Calcitriol upregulated autophagy levels in the injured spinal cord. (a) Transmission electron microscopy showed autophagosomes (black arrowheads) in the calcitriol group (original magnification $\times 7000$ and $\times 20000$ ). (b) Protein expression of LC3-II, Beclin1 and p62 expression in the injured spinal cords of the three groups. GAPDH was used as the loading control and for band density normalisation. (c-e) The optical density analysis of LC3-II, Beclin1 and p62 proteins in the three groups. Data were expressed as the mean \pm s.e.m. ( $n=6$ per group. ${ }^{*} P<0.05$ vs control group, ${ }^{\#} P<0.05$ vs SCl group; The least significant difference test).

the level of cell apoptosis in many diseases. Previous studies have shown that Bcl2 exerts an antiapoptotic effect, ${ }^{15}$ whereas Bax participates in the induction of apoptosis; ${ }^{16}$ activation of C-caspase3 is seen as a hallmark of apoptotic cell death, acting as the final executor of apoptosis. ${ }^{17}$ In the present study, western blotting analyses indicated lower levels of both C-caspase3 and Bax, whereas expression of $\mathrm{Bcl} 2$ was increased, in the calcitriol group relative to the SCI group. Furthermore, both immunohistochemistry for C-caspase3 and TUNEL staining showed significant decreases in the number of apoptosis-positive cells in the spinal cord lesions of calcitriol-treated rats. Therefore, we concluded that calcitriol can inhibit apoptosis in the damaged spinal cord.

MDA is the end product of lipid peroxidation, and the content of MDA is an indicator of free radical levels. ${ }^{18}$ GSH is a major intracellular antioxidant that evades free radicals and catalyses the dismutation of superoxide anions. ${ }^{19}$ The level of GSH reflects the ability to scavenge toxic free radicals. ${ }^{20}$ SOD is an extreme defence against oxygen-free radicals and is an indicator of antioxidant activity with the function of clearing $\mathrm{O}_{2}{ }^{-}$and preventing tissue injury by toxic oxygen-free radicals. ${ }^{21}$ Therefore, SOD activity and the levels of GSH and MDA are important biomarkers of oxidative stress status. Calcitriol has been shown to provide neuroprotection against ischaemia-reperfusion injury by decreasing the level of oxidative stress in the rat hippocampus. ${ }^{22}$ In the present study, we found that SOD activity and GSH content were markedly increased in the calcitriol group compared with controls, whereas the MDA level was decreased. Therefore, we verified that calcitriol suppresses oxidative stress in the damaged SCI. 
a

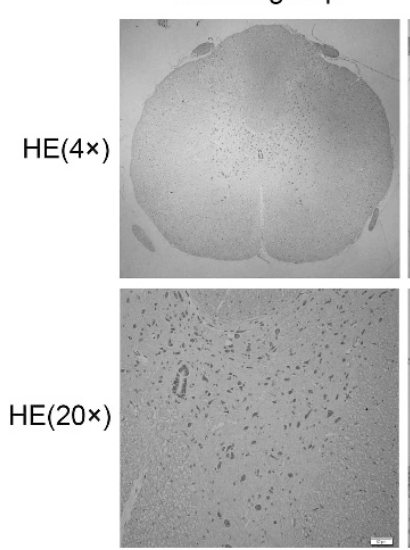

b

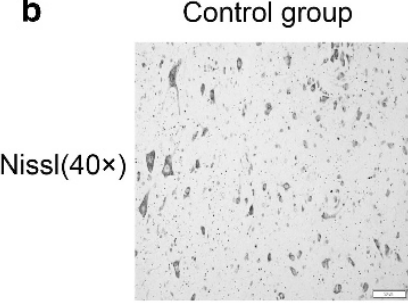

$\mathrm{SCl}$ group
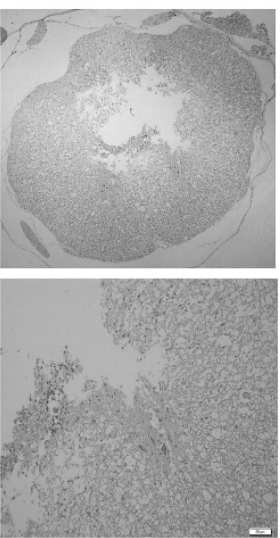

$\mathrm{SCl}$ group

\section{Calcitiol group}
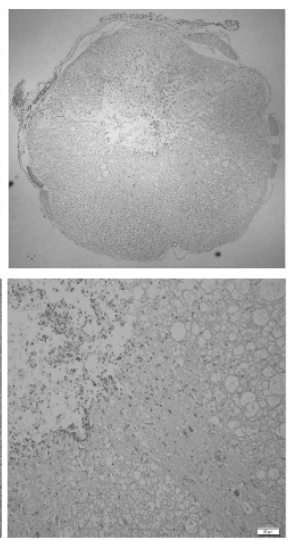

Calcitiol group

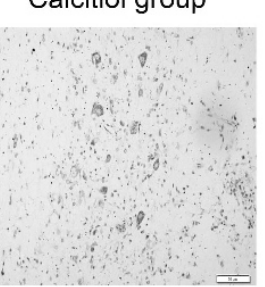

\section{c}

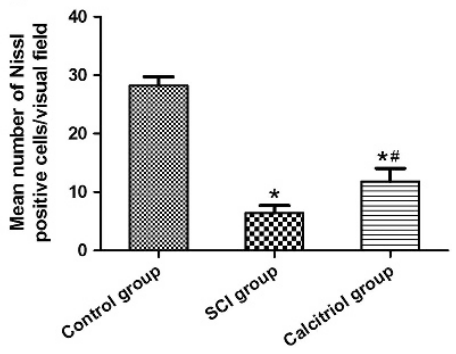

Figure 4 Calcitriol reduces histological damage after SCl. (a) Haematoxylin and eosin (H\&E) staining of the injured spinal cords in the three groups to evaluate histological changes on day 7 day after SCl in the Control, SCl and Calcitriol groups (original magnification $\times 40$ and $\times 200$ ). (b) Nissl staining to assess the loss of neurons in the Control, $\mathrm{SCl}$ and Calcitriol groups (original magnification $\times 400$ ), respectively. (c) The bar graph shows numbers of Nissl-positive cells in the spinal cord lesions in the three groups. Data are expressed as means \pm s.e.m. ( $n=6$ per group. ${ }^{*} P<0.05$ vs control group, $\# P<0.05$ vs $\mathrm{SCl}$ group; The least significant difference test).
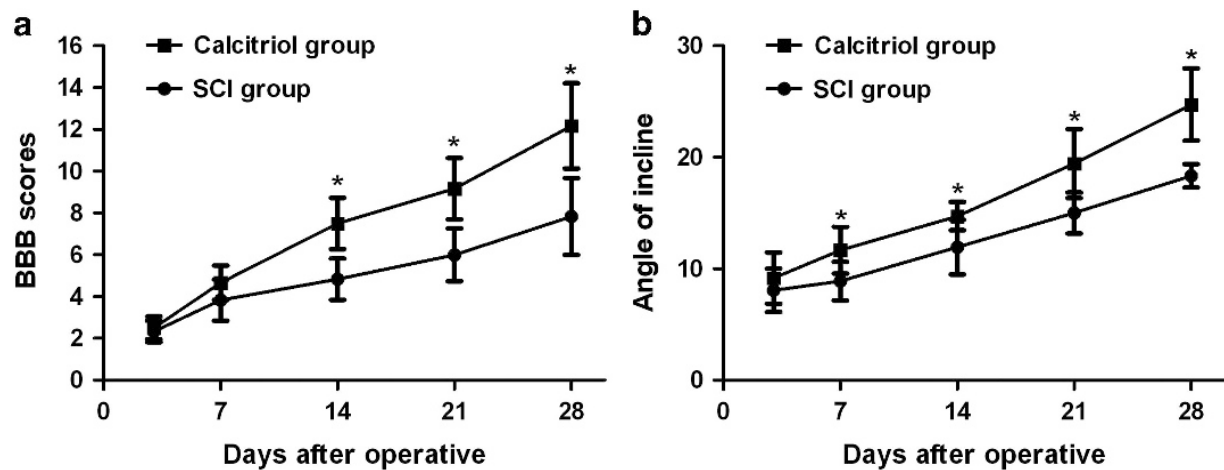

Figure 5 Calcitriol improved functional recovery of rats after SCl. (a) The Basso, Beattie and Bresnahan (BBB) scores of rats in the Control group, the SCl group and the Calcitriol group on days 3, 7, 14, 21 and 28 after contusion. (b) The inclined plane test scores of these three groups at 3, 7, 14, 21 and 28 days after contusion. Data are expressed as means \pm s.d. ( $n=6$ per group. ${ }^{*} P<0.05 \mathrm{vs} \mathrm{SCl}$ group; The least significant difference test).

Autophagy is essential for survival of cells under conditions of metabolic stress, and it is an intracellular degradation process involved in protein homeostasis. However, autophagy is also a specific type of programmed cell death. ${ }^{23}$ Although the role of autophagy in SCI is controversial, increasing numbers of investigations have demonstrated autophagy in rats and the feasibility of manipulating its expression to improve functional outcomes. ${ }^{24}$ Over the past few years, calcitriol has been reported to upregulate the level of autophagy-that is, it can induce autophagy in the SH-SY5Y neuroblastoma cell line as a model of Parkinson's disease. ${ }^{7}$ In addition, calcitriol shows biochemical protective effects in the 1-methyl-4-phenyl-1,2,3,6-tetrahydropyridine mouse model of Parkinson's disease through induction of autophagy. ${ }^{25}$ The present study showed the levels of LC3-II and Beclin1 proteins, representing increased autophagy flux, but the level of p62 protein, which was degraded through the autophagy-lysosomal pathway, decreased in the SCI rats treated with calcitriol compared with the SCI group. Therefore, calcitriol can upregulate the level of autophagy in the damaged spinal cord. It has been confirmed that promotion of autophagy confers a neuroprotective function in SCI. ${ }^{26}$ Moreover, oxidative stress injury can be relieved by autophagy, whereas apoptosis can be inhibited by the activation of autophagy. Therefore, the effects of calcitriol on attenuating oxidative stress and 
decreasing apoptosis after SCI in rats may be mediated through activation of autophagy.

\section{CONCLUSION}

In summary, our investigation demonstrated that locomotor recovery after SCI in rats showed significant improvements with calcitriol treatment, as confirmed by decreases in oxidative stress, inhibition of apoptosis and promotion of autophagy.

\section{DATA ARCHIVING}

There were no data to deposit.

\section{CONFLICT OF INTEREST}

The authors declare no conflict of interest.

\section{ACKNOWLEDGEMENTS}

This study was supported by National Natural Science Foundation of China (81401871, 81401162 and 81572227) and Zhejiang Medical Science Foundation (2013KYA127).

1 Pickett GE, Campos-Benitez M, Keller JL, Duggal N. Epidemiology of traumatic spinal cord injury in Canada. Spine (Phila Pa 1976) 2006; 31: 799-805.

2 Zhang HY, Wang ZG, Wu FZ, Kong XX, Yang J, Lin BB et al. Regulation of autophagy and ubiquitinated protein accumulation by bFGF promotes functional recovery and neural protection in a rat model of spinal cord injury. Mol Neurobiol 2013; 48: 452-464.

3 Chen HC, Hsu PW, Tzaan WC, Lee AW. Effects of the combined administration of vitamins $C$ and $E$ on the oxidative stress status and programmed cell death pathways after experimental spinal cord injury. Spinal Cord 2014; 52: 24-28.

4 Fleet JC, DeSmet M, Johnson R, Li Y. Vitamin D and cancer: a review of molecular mechanisms. Biochem J 2012; 441: 61-76; Review.

5 Giansanti V, Torriglia A, Scovassi Al. Conversation between apoptosis and autophagy: "Is it your turn or mine?". Apoptosis 2011; 16: 321-333.

6 Kanno H, Ozawa H, Sekiguchi A, Itoi E. Spinal cord injury induces upregulation of Beclin 1 and promotes autophagic cell death. Neurobiol Dis 2009; 33: 143-148.

7 Tang P, Hou H, Zhang L, Lan X, Mao Z, Liu D et al. Autophagy reduces neuronal damage and promotes locomotor recovery via inhibition of apoptosis after spinal cord injury in rats. Mol Neurobiol 2014; 49: 276-287.

8 Sezgin G, Ozturk G, Guney S, Sinanoglu O, Tuncdemir M. Protective effect of melatonin and 1,25-dihydroxyvitamin D3 on renal ischemia-reperfusion injury in rats. Ren Fail 2013; 35: 374-379.
9 Jang W, Park HH, Lee KY, Lee YJ, Kim HT, Koh SH. 1,25-dyhydroxyvitamin D3 attenuates L-DOPA-induced neurotoxicity in neural stem cells. Mol Neurobiol 2015; 51: 558-570.

10 Jang W, Kim HJ, Li H, Jo KD, Lee MK, Song SH et al. 1,25-Dyhydroxyvitamin D(3) attenuates rotenone-induced neurotoxicity in SH-SY5Y cells through induction of autophagy. Biochem Biophys Res Commun 2014; 451: 142-147.

11 Basso DM, Beattie MS, Bresnahan JC. A sensitive and reliable locomotor rating scale for open field testing in rats. J Neurotrauma 1995; 12: 1-21.

12 Perrin FE, Boniface G, Serguera C, Lonjon N, Serre A, Prieto M et al. Grafted human embryonic progenitors expressing neurogenin-2 stimulate axonal sprouting and improve motor recovery after severe spinal cord injury. PLOS ONE 2010; 5: e15914.

13 Hong Z, Chen H, Hong H, Lin L, Wang Z. TSP-1 expression changes in diabetic rats with spinal cord injury. Neurol Res 2009; 31: 878-882.

14 Fu J, Xue R, Gu J, Xiao Y, Zhong H, Pan X et al. Neuroprotective effect of calcitriol on ischemic/reperfusion injury through the NR3A/CREB pathways in the rat hippocampus. Mol Med Rep 2013; 8: 1708-1714.

15 Yang J, Liu X, Bhalla K, Kim CN, Ibrado AM, Cai J et al. Prevention of apoptosis by Bcl-2: release of cytochrome c from mitochondria blocked. Science 1997; 275: 1129-1132.

16 Wei MC, Zong WX, Cheng EH, Lindsten T, Panoutsakopoulou V, Ross AJ et al. Proapoptotic BAX and BAK: a requisite gateway to mitochondrial dysfunction and death. Science 2001; 292: 727-730.

17 Min JJ, Huo XL, Xiang LY, Qin $Y Q$, Chai $K Q$, Wu B et al. Protective effect of DI-3n-butylphthalide on learning and memory impairment induced by chronic intermittent hypoxia-hypercapnia exposure. Sci Rep 2014; 4: 5555.

18 Yue C, Chen J, Hou R, Liu J, Li X, Gao Z et al. Effects of selenylation modification on antioxidative activities of Schisandra chinensis polysaccharide. PloS ONE 2015; 10: e0134363.

19 Li H, Jia Z, Li G, Zhao X, Sun P, Wang J et al. Neuroprotective effects of exendin-4 in rat model of spinal cord injury via inhibiting mitochondrial apoptotic pathway. Int J Clin Exp Pathol 2015; 8: 4837-4843.

20 Ueda J, Tsuchiya Y, Ozawa T. Relationship between effects of phenolic compounds on the generation of free radicals from lactoperoxidase-catalyzed oxidation of $\mathrm{NAD}(\mathrm{P}) \mathrm{H}$ or GSH and their DPPH scavenging ability. Chem Pharm Bull (Tokyo) 2001; 49: 299-304.

21 Carda AP, Marchi KC, Rizzi E, Mecawi AS, Antunes-Rodrigues J, Padovan CM et al. Acute restraint stress induces endothelial dysfunction: role of vasoconstrictor prostanoids and oxidative stress. Stress 2015; 18: 1-11.

22 Kajta M, Makarewicz D, Zieminska E, Jantas D, Domin H, Lason W et al. Neuroprotection by co-treatment and post-treating with calcitriol following the ischemic and excitotoxic insult in vivo and in vitro. Neurochem Int 2009; 55: 265-274.

23 Criollo A, Maiuri MC, Tasdemir E, Vitale I, Fiebig AA, Andrews D et al. Regulation of autophagy by the inositol trisphosphate receptor. Cell Death Differ 2007; 14: 1029-1039.

24 Chen HC, Fong TH, Hsu PW, Chiu WT. Multifaceted effects of rapamycin on functional recovery after spinal cord injury in rats through autophagy promotion, anti-inflammation, and neuroprotection. J Surg Res 2013; 179: e203-e210.

$25 \mathrm{Li} \mathrm{H}$, Jang W, Kim HJ, Jo KD, Lee MK, Song SH et al. Biochemical protective effect of 1,25-dihydroxyvitamin D3 through autophagy induction in the MPTP mouse model of Parkinson's disease. Neuroreport 2015; 26: 669-674.

26 Wang ZY, Liu WG, Muharram A, Wu ZY, Lin JH. Neuroprotective effects of autophagy induced by rapamycin in rat acute spinal cord injury model. Neuroimmunomodulation 2014; 21: 257-267. 